\section{Measurements of doses from photon beam irradiation and scattered neutrons in an anthropomorphic phantom model of prostate cancer: a comparison between 3DCRT, IMRT and tomotherapy}

\begin{abstract}
Introduction. The rapid development of new radiotherapy technologies, such as intensity modulated radiotherapy (IMRT) or tomotherapy, has resulted in the capacity to deliver a more homogenous dose in the target. However, the higher doses associated with these techniques are a reason for concern because they may increase the dose outside the target. In the present study, we compared 3DCRT, IMRT and tomotherapy to assess the doses to organs at risk (OARs) resulting from photon beam irradiation and scattered neutrons. Material and methods. The doses to OARs outside the target were measured in an anthropomorphic Alderson phantom using thermoluminescence detectors (TLD 100) ${ }^{6} \mathrm{Li}(7.5 \%)$ and ${ }^{7} \mathrm{Li}(92.5 \%)$. The neutron fluence rate $\left[\mathrm{cm}^{-2} \cdot \mathrm{s}^{-1}\right]$ at chosen points inside the phantom was measured with gold foils $(0.5 \mathrm{~cm}$ diameter, mean surface density of $0.108 \mathrm{~g} / \mathrm{cm}^{3}$ ). Results. The doses [Gy] delivered to the OARs for 3DCRT, IMRT and tomotherapy respectively, were as follows: thyroid gland $(0.62 \pm 0.001$ vs. $2.88 \pm 0.004$ vs. $0.58 \pm 0.003)$; lung $(0.99 \pm 0.003$ vs. $4.78 \pm 0.006$ vs. $0.67 \pm 0.003)$; bladder $(80.61 \pm 0.054$ vs. $53.75 \pm 0.070$ vs. $34.71 \pm 0.059)$; and testes $(4.38 \pm 0.017$ vs. $6.48 \pm 0.013$ vs. $4.39 \pm 0.020)$. The neutron dose from 20 MV X-ray beam accounted for $0.5 \%$ of the therapeutic dose prescribed in the PTV. The further from the field edge the higher the contribution of this secondary radiation dose (from $8 \%$ to $\sim 45 \%$ ). Conclusion. For tomotherapy, all OARs outside the therapeutic field are well-spared. In contrast, IMRT achieved better sparing than 3DCRT only in the bladder. The photoneutron dose from the use of high-energy X-ray beam constituted a notable portion $(0.5 \%)$ of the therapeutic dose prescribed to the PTV.
\end{abstract}

Keywords: 3DCRT $\bullet$ IMRT $\bullet$ low doses $\bullet$ neutron doses $\bullet$ thermoluminescent detectors (TLD) $\bullet$ tomotherapy

A. Kowalik ${ }^{凶}$, M. Skórska, M. Adamczyk, E. Konstanty

Department of Medical Physics,

Greater Poland Cancer Centre,

15 Garbary Str., 61-866 Poznan, Poland,

Tel.: +48 618850552 , +48 61885 0550,

E-mail: anna.nkowalik@gmail.com

J. Malicki, T. Piotrowski

Department of Medical Physics,

Greater Poland Cancer Centre,

15 Garbary Str., 61-866 Poznan, Poland

and Department of Electroradiology,

University of Medical Sciences,

Poznan, Poland

W. Jackowiak

Department of Radiotherapy I,

Greater Poland Cancer Centre,

15 Garbary Str., 61-866 Poznan, Poland

K. Polaczek-Grelik

Department of Medical Physics,

University of Silesia,

4 Uniwersytecka Str., 40-007 Katowice, Poland

Received: 7 July 2015

Accepted: 19 September 2016

\section{Introduction}

Many modern radiotherapy techniques have been developed in recent decades in response to the need to increase the radiation dose to the tumour, while delivering the radiation therapy as accurately as possible. Such advanced techniques include intensity modulated radiotherapy (IMRT), volumetric modulated arc therapy (VMAT), stereotactic body radiation therapy (SBRT), and tomotherapy (TT), among others.

While it is accepted that these treatments deliver more accurate radiation, there are concerns regarding the doses delivered to the nearby organs at risk (OARs). Moreover, the high energy levels used in these treatments may result in significant scatter irradiation. For this reason, it is essential to measure and calculate these low doses; however, doing so presents many challenges [1-4], primarily because the treatment planning systems (TPS) do not calculate doses outside the planning target volume (PTV) more than a few centimetres from the edge of the radiation field. According to the International Commission on Radiological Protection (ICRP), it is necessary to estimate doses outside the PTV from 
all sources in order to assess total stochastic risks, and thus adequately justify radiation exposure in patients treated with radiotherapy (RT) [5].

Numerous studies have evaluated the radiation doses in a body during IMRT and the conventional three-dimensional conformal radiotherapy (3DCRT) [6-10], although fewer have done so for tomotherapy. We carried out the present study in which we compared 3DCRT, IMRT and tomotherapy to assess the doses received by the organs at risk (OARs) resulting from photon beam irradiation and scattered neutrons. The study was performed in an anthropomorphic phantom in a prostate cancer model, with dose measured with lithium fluoride chips (TLD 100).

\section{Material and methods}

\section{Treatment plan preparation}

For measurements of low doses in the body, an anthropomorphic phantom with special thermoluminescent dosimeter (TLD) inserts were prepared. The thickness of each layer in the Alderson phantom is $2.5 \mathrm{~cm}$ and the distance between the dosimetric points is $3 \mathrm{~cm}$, thus allowing for an accurate determination of doses in the selected organs. The doses in OARs located outside the target were measured in the anthropomorphic Alderson phantom using thermoluminescence detectors (TLD) $100\left({ }^{6} \mathrm{Li}(7.5 \%)\right.$ and ${ }^{7} \mathrm{Li}(92.5 \%)$ ); (Harshaw Chemical Company).

In the first phase, the computed tomography (CT) scans were acquired. Subsequently, the clinical target volumes (CTV) were contoured for the prostate (CTV1), lymph nodes (CTV2) and OARs (bladder, rectum, lung, thyroid and testes). The target consisted of the whole pelvic lymph nodes and the prostate gland with seminal vesicles.

In the next stage, three treatment plans were calculated for each of the techniques: 3DCRT (20 MV), IMRT (20 MV) and tomotherapy (6 MV). The dose prescription for each technique was $2 \mathrm{~Gy}$ per fraction up to the total dose of $76 \mathrm{~Gy}$. These energies were used according to the standard protocol for patients with prostate cancer at our institution. For organs inside or close to the treatment fields (rectum, bladder, testes), the doses were read from the TPS. However, the

a

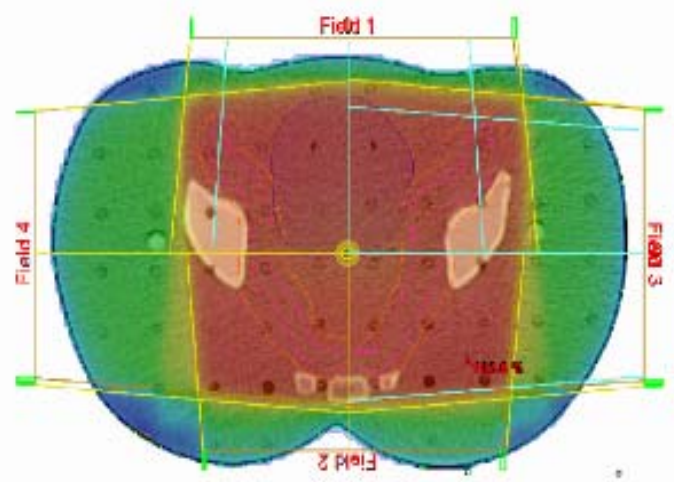

Table 1. The parameters for the tomotherapy plan

\begin{tabular}{lc}
\hline Field width & $2.5 \mathrm{~cm}$ \\
Pitch & 0.287 \\
Planning modulation & 2.700 \\
$\quad$ factor & FINE $(0.254 \mathrm{~cm} \times 0.254 \mathrm{~mm})$ \\
\hline
\end{tabular}

readout of doses for thyroid and lung from the TPS was not possible because commercial systems do not calculate doses at such long distances outside of the target volume.

The aim of each treatment plan was to provide therapeutic dose of 2 Gy using three different techniques, with maximum protection of organs at risk. All parameters of the treatment plans were applied according to the protocol used in our institute for patients with prostate cancer. The treatment plans for 3DCRT technique consisted of four beams - box technique $(0,90,180$ and 270$)$. The total number of Monitor Units (MU) was 163 per fraction. For IMRT, the dose distribution was prepared using seven coplanar beams with the field geometry of $0^{\circ}, 50^{\circ}$, $105^{\circ}, 145^{\circ}, 215^{\circ}, 255^{\circ}$ and $300^{\circ}$. The total number of MUs was 779 per fraction. The tomotherapy plan was generated with the Hi-Art Treatment Planning System (version 4.0.4.17). Based on results from the Skórska et al. [11] study, we used the treatment planning parameters presented in Table 1 . The actual modulation factor was 1.95 . The dose was calculated using a superposition/convolution algorithm with a fine dose calculation grid $(0.254 \mathrm{~cm} \times 0.254 \mathrm{~mm} \times$ $0.3 \mathrm{~cm}$ ). The plan was optimized to reduce the dose to the OARs to a minimum, while the dose to the PTV was maintained in accordance with the ICRU 83 Report [12]. The irradiation time was $407.5 \mathrm{~s}$ and the expected number of MUs was 5863 [13]. Figures 1 and 2 show the dose distribution in the phantom for plans 3DCRT, IMRT and tomotherapy.

\section{Dose measurements}

A total of 100 TLDs per treatment modality were calibrated at $1 \mathrm{~Gy}$. Of these, 73 were chosen for measurements, with a calibration factor below 3\%. This means that the difference between different calibration measurements was not more than 3\% [14].

b

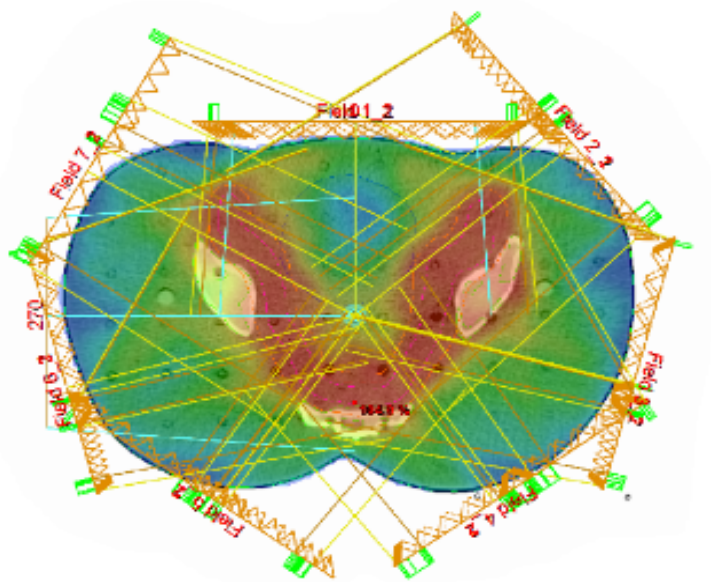

Fig. 1. 3DCRT (a) and IMRT (b) plan for anthropomorphic phantom for prostate gland and lymph nodes. 


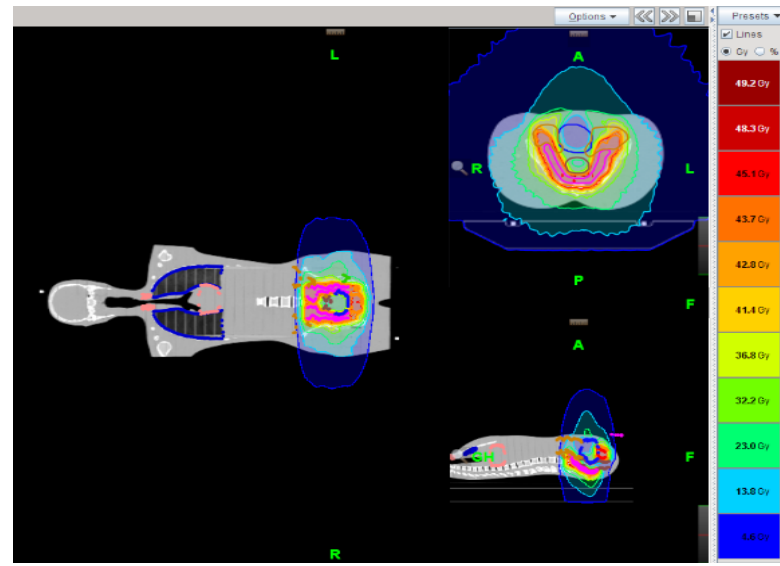

Fig. 2. Tomotherapy plans for anthropomorphic phantom.

\section{Neutron measurements}

Neutron fluence rate $\left[\mathrm{cm}^{-2} \cdot \mathrm{s}^{-1}\right]$ at chosen points inside the Rando ${ }^{\circledast}$ phantom was measured in terms of neutron activation analysis (NAA) with the use of gold foils in the shape of discs that were $0.5 \mathrm{~cm}$ in diameter and having a mean surface density of $0.108 \mathrm{~g} / \mathrm{cm}^{3}$. The induced activity of ${ }^{198} \mathrm{Au}$, measured with the semiconductor gamma spectrometer in terms of net area under the peak $411 \mathrm{keV}$, was directly used to obtain the slow neutron fluence rate values. On the basis of an additional (without an anthropomorphic phantom) measurement of thermal neutron fluence rate $\Phi_{t h}$ in the linac isocenter, the quantity of neutron source strength $Q\left[\mathrm{~s}^{-1}\right]$ was estimated using relation (1). Subsequently, the fast component, which contains direct and scattered fractions of neutron flux, was calculated using Eq. (2) at a distance of $r[\mathrm{~cm}]$ from the beam axis, where OARs were located with respect to the therapeutic plan isocenter and for the surface $S\left[\mathrm{~cm}^{2}\right]$ of the room walls of the linac bunker.

$$
\begin{gathered}
Q=\frac{1.26 \Phi_{\text {th }}}{S} \\
C_{\mathrm{F}-\mathrm{D}}=\frac{4.4 \times 10^{9}}{E_{\mathrm{avg}}^{0.735}}\left[\frac{\mathrm{n} / \mathrm{cm}^{2}}{\mathrm{~Sv}}\right]
\end{gathered}
$$

Table 2. Mean doses calculated in TPS for the bladder for 3DCRT, IMRT and TT techniques per 1 fraction

\begin{tabular}{lccc}
\hline \multirow{2}{*}{ Part of the bladder } & 3DCRT & IMRT & TT \\
\cline { 2 - 4 } \multicolumn{1}{c}{ Mean dose [Gy] } & Mean dose [Gy] & Mean dose [Gy] \\
\hline Top & 2.024 & 1.061 & 0.970 \\
Front wall & 2.016 & 1.107 & 0.941 \\
Back wall & 2.006 & 1.311 & 1.060 \\
Centre & 2.000 & 1.250 & 0.995 \\
Down & 1.990 & 1.404 & 1.103 \\
Mean dose & 2.004 & 1.245 & 1.029 \\
\hline
\end{tabular}

Table 3. The results of measurements of out-of-field irradiation for 3DCRT, IMRT and TT

\begin{tabular}{lrrr}
\hline \multirow{2}{*}{ Localization } & \multicolumn{1}{c}{ IMRT } & \multicolumn{1}{c}{ 3DCRT } & \multicolumn{1}{c}{ TT } \\
\cline { 2 - 4 } & Mean dose [Gy] & Mean dose $[\mathrm{Gy}]$ & Mean dose [Gy] \\
\hline Thyroid & $2.88 \pm 0.004$ & $0.62 \pm 0.001$ & $0.58 \pm 0.003$ \\
Lungs & $4.76 \pm 0.006$ & $0.99 \pm 0.003$ & $0.67 \pm 0.003$ \\
Bladder & $53.75 \pm 0.070$ & $80.60 \pm 0.054$ & $34.71 \pm 0.059$ \\
Testes & $6.48 \pm 0.013$ & $4.37 \pm 0.017$ & $4.38 \pm 0.020$ \\
\hline
\end{tabular}

Neutron dose equivalent at each measuring point (the same as for photon dose measurements) was determined using fluence-to-dose conversion coefficients $\left(C_{\mathrm{F}-\mathrm{D}}\right)$. These were calculated from Eq. (3) involving the average neutron energy $\left(E_{\text {avg }}\right)$, adopted from AAPM Report 19 [15], separately for slow and fast components of neutron flux. Based on the analysis of neutron spectra given in the literature, [16-18] the average neutron energy were taken as $0.2 \mathrm{eV}$ and $0.5 \mathrm{MeV}$ for slow and fast components, respectively. All quantities connected with neutron radiation were referred to the therapeutic dose of 1 Gy deposited in the target volume [19-23].

$$
\Phi_{\text {fast }}\left[\mathrm{cm}^{-2} \cdot \mathrm{s}^{-1}\right]=\frac{Q}{4 \pi r^{2}}+\frac{5.4 Q}{S}
$$

\section{Results}

\section{Mean dose to the bladder using TPS}

The bladder is the organ located closest to the PTV, and therefore, it is possible to measure the dose using the TPS. Notable differences among the three RT techniques were observed for the bladder volume included in the therapeutic field. Use of the 3DCRT technique resulted in larger doses to the bladder vs. IMRT, as shown in Table 2, which shows the mean dose to the various bladder regions for 3DCRT, IMRT and tomotherapy (TT).

\section{Dose measurements}

Table 3 shows the comparison of out-of-field doses between 3DCRT, IMRT and tomotherapy. As shown in Table 3, the scattered radiation from the patient predominates in the region near the therapeutic field. However, as the distance from the field edge increases, the radiation scattered by the collimator, the transmission through the collimator and machine leakage becomes more predominant [24]. A higher beam energy leads to an increase in the out-of-field dose. The effect is enhanced in the proximity of the 
Table 4. The effective estimated out-of-field neutron dose for prostate IMRT

\begin{tabular}{|c|c|c|c|c|}
\hline \multirow{2}{*}{ Localization } & \multicolumn{2}{|c|}{ Effective dose $[\mathrm{mSv} / \mathrm{Gy}]$} & \multirow{2}{*}{$\begin{array}{l}\text { Total neutron effective } \\
\text { dose normalized } \\
\text { to } 76 \text { Gy in PC [mSv] }\end{array}$} & \multirow{2}{*}{$\begin{array}{c}\text { Mean normalized } \\
\text { dose in organ } \\
{[\mathrm{mSv}]}\end{array}$} \\
\hline & Slow neutrons & Fast neutrons & & \\
\hline Pelvis - right side & $0.022 \pm 0.008$ & $6.4 \pm 2.7$ & $6.4 \pm 2.8$ & \multirow{4}{*}{6.201} \\
\hline Pelvis - left side & $0.038 \pm 0.009$ & $6.0 \pm 1.8$ & $6.0 \pm 1.8$ & \\
\hline Right lung - corner & $0.032 \pm 0.010$ & $5.9 \pm 2.3$ & $6.0 \pm 2.3$ & \\
\hline Left lung - corner & $0.026 \pm 0.012$ & $6.1 \pm 3.2$ & $6.1 \pm 3.2$ & \\
\hline Right lung - centre & $0.026 \pm 0.016$ & $5.9 \pm 4.2$ & $6.0 \pm 4.2$ & \multirow{4}{*}{5.832} \\
\hline Left lung - centre & $0.050 \pm 0.014$ & $5.3 \pm 1.9$ & $5.4 \pm 1.9$ & \\
\hline Right lung - top & $0.017 \pm 0.008$ & $5.9 \pm 3.1$ & $5.9 \pm 3.1$ & \\
\hline Left lung - top & $0.023 \pm 0.010$ & $5.7 \pm 3.0$ & $5.7 \pm 3.0$ & \\
\hline Right eye & $0.024 \pm 0.009$ & $5.161 \pm 2.407$ & $5.184 \pm 2.418$ & \multirow{2}{*}{5.189} \\
\hline Left eye & $0.023 \pm 0.010$ & $5.170 \pm 2.540$ & $5.194 \pm 2.552$ & \\
\hline
\end{tabular}

field edge, where the dose drop is much sharper for Neutron dose results

$6 \mathrm{MV}$ photons than for 15 and $20 \mathrm{MV}$ photons.

\section{DCRT}

For 3DCRT, the bladder was located within the radiation field, and therefore, received a dose that was nearly equal to the dose to the prostate. The dose in the thyroid, located approximately $60 \mathrm{~cm}$ from the central beam axis (CAX), was $0.621 \mathrm{~Gy}$, which is about $1 \%$ of the CAX. The dose in the lung equalled $1.3 \%$ of the total dose. In the phantom, the TLDs were located on the surface of lungs, both at peripheral and central parts. The detectors located near the pelvis yielded higher dose measurements than TLDs located in other areas. The mean dose to the bladder with 3DCRT was 80.6 Gy. The testes were located close to the radiation field and received $4.4 \%$ of the total dose.

\section{IMRT}

For IMRT, the bladder was located partially in the field, and received a dose similar to that received by the prostate. The radiation field was larger than in 3DCRT, and consequently the dose to some OARs was higher; however, the dose to the bladder was lower than with 3DCRT (53.7 Gy vs. 80.6 Gy) because it was outside the field of radiation. The total IMRT dose to the thyroid gland was approximately 5 times higher than with 3DCRT (2.88 Gy vs. $0.62 \mathrm{~Gy})$, representing about $4 \%$ of the dose in the PTV. In the lungs, the measured dose was $4.8 \mathrm{~Gy}$. The dose in the testes was 2 Gy larger than that achieved with 3DCRT.

\section{Tomotherapy}

For tomotherapy, the total doses (for complete treatment process to $76 \mathrm{~Gy}$ ) to the thyroid gland, lungs, bladder, and testes were, respectively, 0.6, 0.7, 34.7 and $4.4 \mathrm{~Gy}$.
The neutron effective dose (Table 4) did not vary significantly across the whole body of the phantom for IMRT technique ${ }^{1}$ : the arithmetic mean over the complete results of photoneutron dose estimation is $5.777 \mathrm{mSv} / \mathrm{Gy}$ and the standard deviation of this mean value is 0.127 . The dispersion of the results achieved at particular points across the phantom reaches only $19 \%$. However, the dose tended to decrease as the distance from the central beam axis (CAX) increased, a phenomenon that is explained by the thermalization of neutrons and subsequent capture. The symmetrical (with respect to the long axis of the phantom/therapeutic couch) distribution of neutron fluence can be observed in Fig. 3. This distribution is connected with the specific treatment plan for IMRT technique implemented in the present research. The uncertainty of these values (given in Table 4) are high because they are affected by several factors, the most important of which is related to the net peak area dependent on the spectrometer counting statistics (when the activity of detection foil is assigned), and the fluence-to-dose conversion coefficients used (10\%).

\section{Discussion}

Modern radiotherapy systems deliver highly targeted doses. However, because TPS do not calculate doses more than few centimetres outside the PTV, organs outside the field may receive high doses, leading to a multitude of adverse effects. In the present study, we evaluated three common RT modalities - IMRT, SBRT and tomotherapy - in a phantom model to determine the doses from both scattered and direct radiation delivered to the surrounding OARs. The main finding was that tomotherapy provided the best overall sparing of organs located outside the therapeutic field. Surprisingly, 3DCRT achieved better sparing than IMRT for all organs except for bladder. In addition, the photoneutron dose (re-

\footnotetext{
${ }^{1}$ Doses for neutrons were measured in cooperation with another institute. Doses were measured only for IMRT technique, and unfortunately it was not possible to make the measurements for the other two techniques.
} 
a

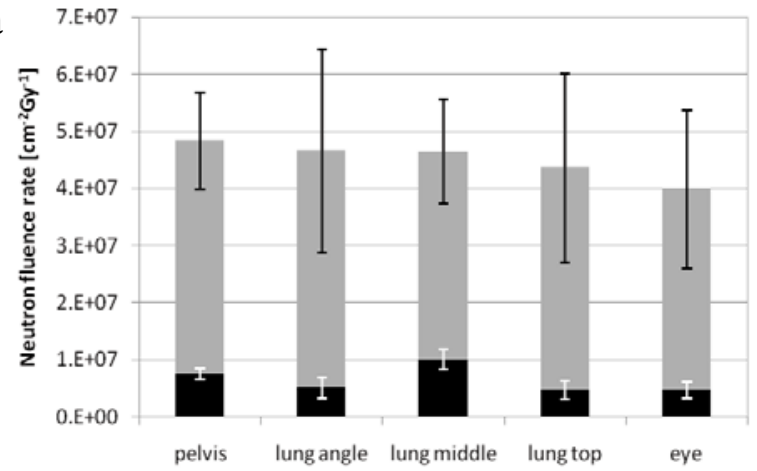

b

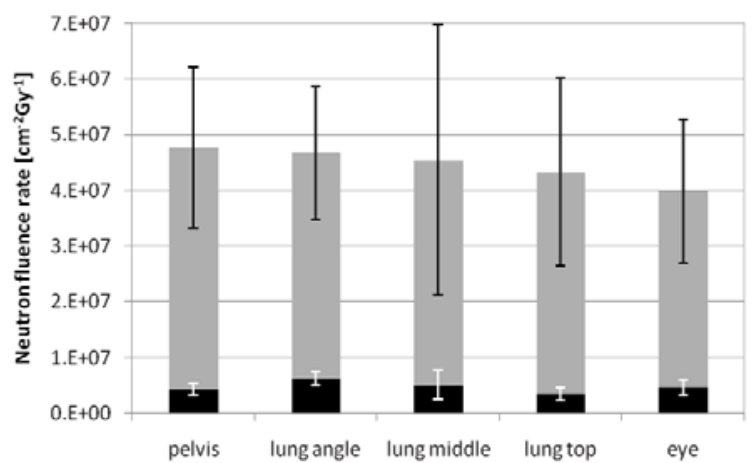

Fig. 3. The distribution of neutron fluence during prostate IMRT normalized to 1 Gy of dose in PTV (a) and of the anthropomorphic phantom (b). The slow (black) and the fast (grey) components are separated, showing the decrease of total neutron fluence when the distance from the isocentre is increased.

sulting from the use of high-energy X-ray beam) constituted approximately $0.5 \%$ of the therapeutic dose prescribed in the PTV.

As expected, doses to the remaining points were higher for IMRT, but the magnitude of doses at the more distant points became quite small. The differences can be explained by considering the different components of the peripheral dose: (1) the linear accelerator head leakage, which affects the entire body equally and is directly proportional to monitor units, (2) the internal scatter, which primarily affects tissues several centimetres from the beam edge and is directly proportional to field size, and (3) the entrance and exit extra-target doses in the plane of beams will expose more tissues to lower doses in the plane of the beams.

Measuring low doses presents many challenges because the spectrum of the scatter radiation is unknown, and detectors must therefore be independent of the energy spectrum. Moreover, it is not possible to verify doses in OARs located outside the therapeutic field in vivo. For this reason, anthropomorphic phantoms such as the Alderson phantom with special TLD inserts are needed. This phantom provides the ideal conditions for dose verification outside the target, and the TLDs are both precise and independent of the energy spectrum.

As discussed previously, the lack of information from TPS regarding doses outside the beam path is problematic. Similarly, the fact that the TPS does not account for neutron radiation, which in modern RT techniques can constitute a significant part of the dose to organs outside the target, presents an additional problem. It is clear that when high-energy X-ray beams are used in radiotherapy treatment, the presence of secondary neutron radiation should also be taken into account. Computer simulations of the spectra of therapeutic photon beams $[25,26]$ have shown that up to $30 \%$ of photons could have energy above the threshold of nuclear reactions $(\gamma$, $n$ ) for heavy elements constituting the accelerator head. These photonuclear interactions are the main source of fast neutrons, produced mostly via giant dipole resonance (GDR) [27]. As the photoneutrons leave the linear accelerator (linac), their energy spectrum becomes moderated. Therefore, in the isocenter, two main components are distinguished: the fast one with an average energy of about
$0.5 \mathrm{MeV}$, and the slow one with the distribution peaked at thermal energy $\left(E_{\mathrm{Av}}=0.025 \mathrm{eV}\right)$ and a tail on the high-energy site $\left(E_{\mathrm{Av}}=0.05 \mathrm{MeV}\right)$, as several studies have shown $[28,29]$. Since the linear energy transfer (LET) and the relative biological effectiveness (RBE) of neutrons depend strongly on their energy, these two main components should be taken into account when evaluating the additional dose from secondary radiation during radiotherapy.

A somewhat surprising finding of this study was that IMRT resulted in worse OAR sparing than 3DCRT. The reasons for this difference may be explained by the longer beam-on time required with IMRT, and the consequent increased exposure to leakage radiation. Moreover, the use of more than four fields resulted in a larger volume of tissue exposed to relatively low doses $[30,31]$. As compared to the conventional 3DCRT, IMRT requires the emission of more MUs to deliver the same dose to PTV. Also, the field size does not remain constant during the whole procedure. These two factors greatly influence the production of photoneutrons, as well as the contribution of fast neutrons (of higher RBE), which is connected with the field size. The effective neutron dose relates also to the depth inside the body (phantom). Multi-directional dose delivery in the IMRT technique causes the geometry of neutron irradiation to be more isotropic. To avoid neutron irradiation of asymmetrical OARs, changes in the treatment plan, such as the use of a different distribution of beam directions (i.e., not symmetrical), could be considered.

The bladder and testes, as accepted, received the highest dose because the OAR in 3DCRT was within the field of radiation and very near the field of radiation for IMRT and tomotherapy. In addition, neutron contamination from high-energy therapeutic beams (such as, the 20 MV IMRT beam) undoubtedly contributed to these doses. In other regions (pelvis, chest and eyes), the effective doses due to photoneutrons were estimated with the NAA method.

\section{Limitations}

The doses in OARs were measured under ideal conditions: there was no movement of the phantom 
during the treatment. In case of a real patient, these doses would be more variable depending on the organ motion or bladder and rectal filling. In the phantom, the dose measurements were clustered around the same range of values due to the stable nature of phantom geometry; in a live patient, this would not be the case, but for the organs placed at a distance of 50-60 cm from the isocenter, the movements can be insignificant.

\section{Conclusion}

Among the three treatment modalities evaluated in the present study, we found that tomotherapy provides the best OAR sparing. Surprisingly, 3DCRT provided better sparing than IMRT for all OARs except the bladder. The further from the field edge the higher the contribution of this secondary radiation dose. The use of lower energies, when feasible, allows avoiding undesirable scatter radiation and its negative effects.

The low doses and secondary radiation can have an impact on the normal tissues and OARs, therefore, more research in this area is needed. Research also requires relation doses in organ at risk to stochastic effects, which are the subject of another work of the authors.

Acknowledgment. The project was funded by a grant from the National Science Centre Poland no. OPUS 2012/01/B/NZ4/03736.

This publication was written under an internal project (Agreement no. 3, 27.09.2015) which was financial supported by the Greater Poland Cancer Centre.

\section{References}

1. Francois, P., Beurtheret, C., \& Dutreix, A. (1988). Calculation of the dose delivered to organs outside the radiation beams. Med. Phys., 15(6), 879-883.

2. Howell, R. M., Scarboro, S. B., Kry, S. F., \& Yaldo, D. Z. (2010). Accuracy of out-of-field dose calculations by a commercial treatment planning system. Phys. Med. Biol., 55(23), 6999-7008.

3. Sheikh-Bagheri, D., \& Rogers, D. W. O. (2002). Monte Carlo calculation of nine megavoltage photon beam spectra using the BEAM code. Med. Phys., 29, 391-402.

4. Schulte, R. W., Rittmann, K. L., Meinass, H. J., \& Rennicke, P. (1996). Radiation dose in critical organs due to non-coplanar irradiation of the hypophysis. Strahlenther. Onkol., 172(9), 501-506.

5. ICRP. (1991). 1990 Recommendations of the International Commission on Radiological Protection. Ann. ICRP, 21(1/3).

6. Kaderka, R., Schardt, D., Durante, M., Berger, T., Ramm, U., Licher, J., \& La Tessa, C. (2012). Out-offield dose measurements in a water phantom using different radiotherapy modalities. Phys. Med. Biol., 57, 5059-5074.

7. Kase, K. R., Syensson, G. K., Wolbarst, A. B., \& Marks, M. A. (1983). Measurements of dose from secondary radiation outside a treatment field. Int. J. Radiat. Oncol. Biol. Phys., 9(8), 1177-1183.
8. Kry, S. F., Salehpour, M., Followill, D. S., Stovall, M., Kuban, D. A., White, R. A., \& Rosen, I. I. (2005). Outof-field photon and neutron dose equivalents from step-and-shoot intensity-modulated radiation therapy. Int. J. Radiat. Oncol. Biol. Phys., 62 (4), 1204-1216.

9. Peszynska-Piorun, M., Malicki, J., \& Golusinski W. (2012). Doses in organs at risk during head and neck radiotherapy using IMRT and 3D-CRT. Radiol. Oncol., 46(4), 328-336.

10. Van den Heuvel, F., Defraene, G., Crijns, W., \& Bogaerts, R. (2012). Out-of-field contributions for IMRT and volumetric modulated arc therapy measured using gafchromic films and compared to calculations using a superposition/convolution based treatment planning system. Radiother. Oncol., 105(1), 127-132.

11. Skórska, M., \& Piotrowski, T. (2013). Optimization of treatment planning parameters used in tomotherapy for prostate cancer patients. Phys. Med., 29(3), 273-285.

12. ICRU. (2010). Prescribing, recording, and reporting intensity-modulated photon-beam therapy (IMRT). Washington: International Commission on Radiation Units and Measurements. (ICRU Report 83).

13. Mackie, T. R., Holmes, T., Swerdloff, S., Reckwerdt, P., Deasy, J. O., Paliwal, B., \& Kinsella, T. (1993). Tomotherapy: a new concept for the delivery of dynamic conformal radiotherapy. Med. Phys., 20, 1709-1719.

14. Harris, C. K., Elson, H. R., Lamba, M. A., \& Foster, A. E. (1997). Comparison of effectiveness of thermoluminescent crystals LiF: $\mathrm{Mg}, \mathrm{Ti}$, and $\mathrm{LiF}: \mathrm{Mg}, \mathrm{Cu}, \mathrm{P}$ for clinical dosimetry. Med. Phys., 24(9), 1527-1529.

15. Nath, R., Boyer, A. L., La Riviere, P., McCall, R., \& Price, K. (1986). Neutron measurements around high energy X-ray radiotherapy machines. New York: American Association of Physists in Medicine. (AAPM Report No. 19).

16. Facure, A., Falcão, R. C., Silva, A. X., Crispim, V. R., \& Vitorelli, J. C. (2005). A study of neutron spectra from medical linear accelerators. Appl. Radiat. Isot., $62,69-72$.

17. Kralik, M., \& Turek, K. (2004). Characterisation of neutron fields around high-energy X-ray radiotherapy machines. Radiat. Prot. Dosim., 110(1/4), 503-507.

18. Vega-Carrillo, H. R., Ortiz-Hernandez, A., HernandezDavila, V. M., Hernández-Almaraz, B., \& Rivera Montalvo, T. (2010). $\mathrm{H}^{*}(10)$ and neutron spectra around linacs. J. Radioanal. Nucl. Chem., 283, 537-540.

19. Harrison, R. M., Wilkinson, M., Shemilt, A., Rawlings, D. J., Moore, M., \& Lecomber, A. R. (2005). Estimating second cancer risk following radiotherapy: organ doses from prostate radiotherapy and concomitant exposures. Biomed. Tech., 50 (Suppl. 1, Pt 1), 768-769.

20. Kry, S., Salehpour, M., Followill, D., Stovall, M., Kuban, D., White, R., \& Rosen, I. I. (2005). The calculated risk of fatal secondary malignancies from intensity-modulated radiation therapy. Int. J. Radiat. Oncol., 62(4), 1195-1203.

21. Stovall, M., Smith, S. A., Langholz, B. M., Boice, J. D., Shore, R. E., Andersson, M., Buchholz, T. A., Capanu, M., Bernstein, L., Lynch, C. F., Malone, K. E., Anton-Culver, H., Haile, R. W., Rosenstein, B. S., Reiner, A. S., Thomas, D. C., Bernstein, J. L., \& WECARE Study Collaborative Group. (2008). Dose to the contra-lateral breast from radiotherapy and risk of second primary breast cancer in the WECARE study. Int. J. Radiat. Oncol., Biol. Phys., 72(4), 1021-1030.

22. Al-Ghamdi, H., Fazal-ur-Rehman, Al-Jarallah, M. I., \& Maalej, N. (2008). Photoneutron intensity with field size around radiotherapy linear accelerator 18-MeV X-ray beam. Radiat. Meas., 43, S495-S499. 
23. Chibani, O., \& Ma, Ch. -M. Ch. (2003). Photonuclear dose calculations for high-energy photon beams from Siemens and Varian linacs. Med. Phys., 30(8), 1990-2000.

24. D’Errico, F., Nath, R., Tana, L., Curzio, G., \& Alberts, W. G. (1998). In-phantom dosimetry and spectrometry of photoneutrons from an $18 \mathrm{MV}$ linear accelerator. Med. Phys., 25(9), 1717-1724.

25. Hall, E. J., \& Wuu, C. (2003). Radiation-induced second cancers: the impact of 3D-CRT and IMRT. Int. J. Radiat. Oncol. Biol. Phys., 56, 83-88.

26. Hernández, T. G., González, A. V., Peidro, J. P., Ferrando, J. V. R., González, L. B., Cabañero, D. G., \& Torrecill, J. L. (2013). Radiobiological comparison of two radiotherapy treatment techniques for high-risk prostate cancer. Rep. Pract. Oncol. Radiother., 18(5), 265-271.

27. Howell, R., Hertel, N. E., Wang, Z., Hutchinson, J., \& Fullerton, G. D. (2006). Calculation of effective dose from measurements of secondary neutron spectra and scattered photon dose from dynamic MLC IMRT for
$6 \mathrm{MV}, 15 \mathrm{MV}$ and $18 \mathrm{MV}$ beam energies. Med. Phys., 33(2), 360-368.

28. Kase, K. R., Mao, X. S., Nelson, W. R., Liu, J. C., Kleck, J. H., \& Elsalim, M. (1998). Neutron fluence and energy spectra around the Varian Clinac 2100C/2300C medical accelerator. Health Phys., $74,38-47$.

29. Kourinou, K. M., Mazonakis, M., Lyraraki, E., Stratakis, J., \& Damilakis, J. (2012). Scattered dose to radiosensitive organs and associated risk for cancer development from head and neck radiotherapy in pediatric patients. Phys. Medica, 29(6), 650-655.

30. Lambrecht, M., Nevens, D., \& Nuyts, S. (2013). Intensity-modulated radiotherapy vs. parotid-sparing 3D conformal radiotherapy. Strahlenther. Onkol., 189(3), 223-229.

31. Leszczyński, W., Ślosarek, K., \& Szlag, M. (2012). Comparison of dose distribution in IMRT and Rapid Arc technique in prostate radiotherapy. Rep. Pract. Oncol. Radiother., 17(6), 347-351. 